\title{
LAS REPERCUSIONES DE LAS REFORMAS AGRARIAS DEL PLAN DE RIEGOS DEL ALTO ARAGÓN EN LA POBLACIÓN DE LA COMARCA ARAGONESA DE LOS MONEGROS
}

\author{
Bouzaida, M.A., y Frutos, L.M. \\ Dpto. de Geografía y Ordenación del Territorio \\ Universidad de Zaragoza
}

\section{RESUMEN}

El regadío es uno de los componentes clave para el desarrollo socioeconómico de una región, sobre todo en la cuenca del Mediterráneo, siendo, además, un elemento fundamental de la estructura del paisaje y una de las variables territoriales más relevante, tanto en términos de ocupación de superficie como de utilización y de consumo de agua. Este último es un recurso natural básico e imprescindible para el funcionamiento de los sistemas ecológicos de la naturaleza y para el desarrollo de las actividades humanas, razones por las cuales las reformas agrarias generadas tras la transformaciones en regadío están concebidas, entre otros objetivos, para asegurar el desarrollo económico y mejorar la calidad de vida de una región, fijando su población. De lo contrario, la salud y el bienestar humano, la seguridad alimentaria, el desarrollo industrial y los ecosistemas ligados a los sistemas hídricos están puestos en peligro. La comarca alto-aragonesa de Los Monegros es un ejemplo muy relevante de ello. Este trabajo analiza la incidencia social de la puesta en regadío en dicha comarca y pretende comprobar si este recurso económico ha permitido fijar población en la zona, mejorando su nivel de vida, tal y como plantean las teorías sobre este tema.

Palabras clave: Aragón, Los Monegros, población, reformas agrarias, Regadío, incidencia social, desarrollo socioeconómico.

\section{SUMMARY}

The irrigation is mainly one of the key components for the socioeconomic development of a region in the Mediterranean basin, being also, a fundamental element in the landscape structure and one of the most outstanding territorial variables, so much in terms of surface occupation, as of use and of water consumption. This last is a basic and indispensable natural resource for the ecological systems and for the human activities progress. That's the reason why the agrarian reforms generated after transformations in irrigated land are conceived among several objectives to assure the economic development and to improve 
the live quality of the region fixing its population. Otherwise, the health and the human well-being, the food security, the industrial development and the ecosystems related to the hydric systems are threatened. The northern aragoneese region of los Monegros is a very outstanding example of it. This work analyse the social incidence of the transformation in irrigated land in this area and try to verify if this economic resource has allowed to fix population there improving its life level, according to the theories planning on this subject.

Key words: Aragon, Los Monegros, population, agrarian reforms, irrigation, social incidence, socio-economic development.

\section{Introducción}

Es evidente que el descenso de la población activa agrícola y el incremento de la ocupada en los servicios parece imparable: según el Libro Blanco Del Agua (Ministerio del Medio Ambiente, 2000), en España la ocupación en el sector agrícola se ha reducido, en apenas quince años, a casi la mitad, pasando de cerca de 2 millones en 1984 a poco más de 1 millón en 1999. Cabe prever que estas tendencias continuarán en los próximos años y que, previsiblemente, en el año 2010 la población agraria ocupada no superará el medio millón de empleos. Además, este retroceso general a nivel nacional, será más intenso en los territorios de la España rural interior y septentrional. No obstante, pese a estas tendencias generales debe señalarse la relativamente elevada dependencia que aún se registra en gran parte del territorio español respecto del sector primario, reflejo de la tradicional vocación agraria de una parte apreciable de la estructura productiva española. Aunque la media de participación de la agricultura y la pesca en el Valor Añadido Bruto total nacional es inferior al $5 \%$, existen provincias donde se supera el $15 \%$.

Así mismo, desde una perspectiva territorial, y según la misma fuente, cabe mencionar también la situación en la que actualmente se encuentran muchos de los núcleos del interior peninsular, que tenderá a agravarse en el futuro. En estos núcleos, al no alcanzarse los umbrales de población requeridos para poder aprovechar las necesarias economías de escala, surgirán problemas para financiar y gestionar eficientemente los servicios básicos, como el de abastecimiento de agua y depuración de los efluentes, entre otros. La intervención pública a tales efectos parece de obligada necesidad si se desea coayuvar a la subsistencia y mejora de la calidad de vida de estos núcleos rurales. En este sentido es un hecho indudable que las generaciones jóvenes sólo estarán dispuestas a continuar en la actividad agrícola si sus expectativas de renta se acercan a las que se les ofrece en otras actividades, y eso no ocurre, pese a las ayudas compensatorias de la Política Agraria Común (PAC). Por otro lado, esta misma política ha provocado abandono de tierras y jubilación anticipada sin ofrecer suficientes alicientes a los jóvenes agricultores para su instalación.

Siguiendo a Gómez et al. (2000), en España, el éxodo rural es consecuencia de la crisis de la agricultura tradicional, lo que se debió, en primer lugar, a la dificultad de introducir en el campo unas técnicas adecuadas de producción y distribución que permitieran el crecimiento y modernización del sector con el sistema existente. En segundo lugar, a la falta de empresarios agrícolas con suficiente formación y recursos para favorecer el cambio; en tercer lugar, a las peculiaridades de la infraestructura agraria, compuesta en buena parte por pequeñas explotaciones familiares, en ocasiones destinadas al autoabastecimiento familiar, o medianas explotaciones con escaso nivel de inversión y, por tanto, de rentabilidad.

Así, a lo largo del siglo XX, y especialmente en las últimas décadas, el campo ha expulsado importantes contingentes de población a causa de la crisis de la agricultura 
tradicional, sobre cuyo tema se ha escrito mucho (Marín Cantalapiedra, 1973; Camarero, 1992; Higueras, 1996; Frutos, 1998), y también de la mecanización creciente y de la falta de alicientes del medio rural y se teme que se mantenga una emigración selectiva por causas socioeconómicas, especialmente en zonas deprimidas en las que se produce una elevada tasa de mortalidad, unos bajos porcentajes de población ocupada, una renta per capita menor, un predominio de la actividad agraria entre su población activa y una huida hacia otros sectores más productivos, prósperos y rentables.

A escala menor, y como numerosos trabajos han puesto de relieve (Ortega, 1979; Bielza, 1987; Faus y Higueras, 1986, 2000; Frutos 1994, 1998), la emigración ha sido una constante histórica en gran parte del territorio de la Comunidad Aragonesa desde el comienzo del siglo XX, lo que ha afectado tanto a las posibilidades de crecimiento y desarrollo de amplios espacios geográficos, como a su estructura demográfica, que se ha visto afectada por el éxodo rural desde una doble dimensión: por una parte, por el descenso paulatino y constante de su población, que alcanza las mayores pérdidas entre los años cincuenta y setenta, y como consecuencia de ello, y teniendo presente que quienes emigran son personas en edad activa y con capacidad de reproducción, se reduce la natalidad y se incrementa la proporción de ancianos, a lo que se suma la mayor esperanza de vida, lo que se resuelve en elevados índices de envejecimiento, bajos índices de natalidad y menor capacidad de cambio.

La modernización de la agricultura se contempla como una de las posibilidades de frenar esta sangría demográfica y, en este marco, se integra la transformación en regadío, como uno de los aspectos de las reformas agrarias, entendidas como un intento de resolver los desajustes sociales y económicos ligados a la estructura de la propiedad y de la explotación, definida por Kostrowicki (1980), en sentido amplio, como «todas las mutaciones de relación agrícola introducidas de modo evolutivo o revolucionario por iniciativa de los poderes públicos o de instituciones privadas». De otra parte, y de modo más preciso, George (1980) entiende que una reforma agraria es el conjunto de operaciones que tienden a transformar la estructura territorial de un Estado o de una región, mediante la modificación de las relaciones sociales, con el fin de asegurar las técnicas de cultivo y el aumento de la producción agrícola y en sentido estricto conlleva una distribución de la tierra y la eliminación de los latifundios. La reforma agraria por consiguiente, es uno de los métodos aceptados para fijar la población, con sus múltiples implicaciones (económicas, sociales, políticas, medioambientales, etc.).

En esta dinámica está la comarca aragonesa de Los Monegros, ubicada en el centro del Valle del Ebro, a caballo entre la provincia de Huesca y la de Zaragoza (Figura 1), que en los años sesenta quedó incluida en la transformación en regadío, dentro del Plan de Riegos del Alto Aragón, cuya finalidad era asegurar los cultivos de cereales de invierno, hacer anuales las cosechas, hasta entonces dependientes de un clima duro y imprevisible, e introducir nuevos cultivos, intentando así mismo evitar el progresivo despoblamiento de la zona, siendo el objetivo principal de estas reformas aumentar los recursos agrícolas, ganaderos e industriales. Por lo tanto, ha habido un proceso de transformación en la comarca, generando un cambio en la distribución de la superficie cultivada, la propiedad, la parcelación y la explotación. En consecuencia, los usos del suelo se han modificado, se ha instalado un nuevo sistema de poblamiento al construir los núcleos de colonización en la zona regada y se ha creado una red de infraestructura de riego y de caminos.

Transcurridas varias décadas desde la puesta en regadío de las tierras monegrinas, en este trabajo se pretende verificar si se ha alcanzado uno de los objetivos de la transformación, que es el freno a la emigración y el mantenimiento e incluso el incremento de la población, al ofrecer nuevas oportunidades de trabajo. En el inicio de los años ochenta 


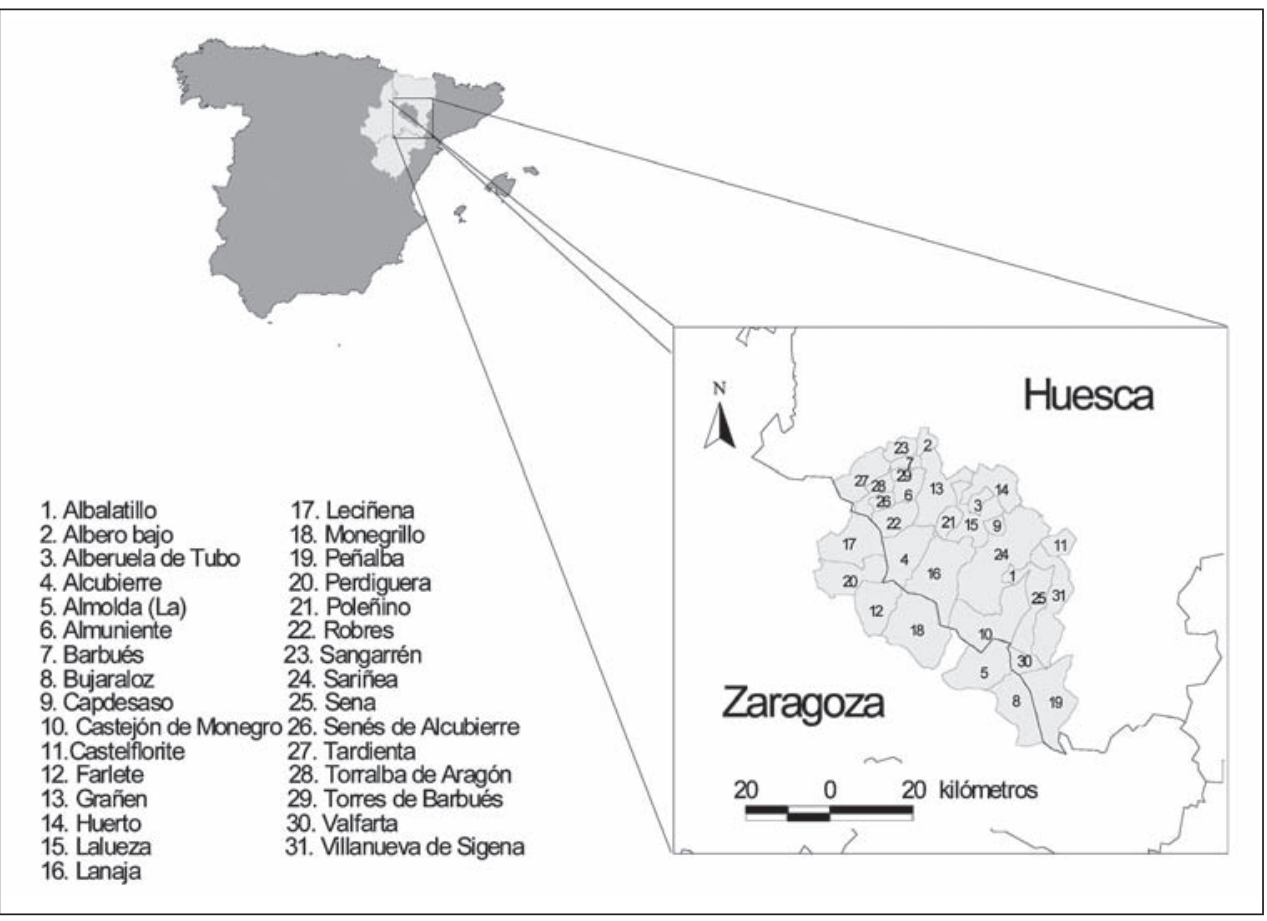

Figura 1. Mapa de ubicación de Los Monegros.

Elaboración propia.

Castelló (1989) puso de manifiesto que estas expectativas no se habían cubierto. Por ello, en las páginas siguientes se analizará lo que ha ocurrido en el transcurro de estas últimas décadas, buscando las causas del comportamiento demográfico de la comarca.

\section{Método y fuentes}

Se parte en este trabajo, como se ha apuntado al principio, de un modelo de desarrollo rural que relaciona la fijación de la población y la mejora socioeconómica de los agricultores con una transformación técnica que, supuestamente, permitirá la creación de riqueza, facilitará las reinversiones diversificadas y creará, por tanto, más puestos de trabajo, tanto en el sector primario como en los demás. Para comprobar dicha hipótesis se ha analizado, en primer lugar, la evolución poblacional de la comarca de Los Monegros a lo largo del siglo XX, haciendo especial hincapié en las variaciones internas entre municipios de secano y de regadío y analizando los índices demográficos. En segundo lugar, se ha indagado en las posibles causas de este comportamiento.

Para ello se han consultado las fuentes bibliográficas y las estadísticas oficiales disponibles, tanto del Instituto Nacional de Estadística (INE), como del Instituto Aragonés de Estadística (IAE), las del ministerio de Agricultura, Pesca y Alimentación (MAPA) y las de las Consejerías de Agricultura y de Industria de la DGA, así como las de otras entidades y organismos para temas concretos. Estas fuentes son las que se citan a continuación: 
— La evolución intercensal de la población desde 1900 hasta 2001.

- Los Nomenclátores desde los años 50.

- Los datos de la población activa y la evolución interanual (1985-2003).

- El mercado laboral en las comarcas aragonesas (2003-2005).

- Los Censos Agrarios desde 1962 hasta 1999.

- Los anuarios estadísticos; y los resultados técnico-económicos de las explotaciones agrícolas en Aragón (2000-2003).

- Los datos de superficie y de aprovechamiento del 1T.

— Los censos de las empresas agroalimentarias del año 2005.

Todos los datos han sido contrastados y elaborados a nivel municipal y comarcal, para lo que fue necesario reconstruirlos desde los años sesenta hasta la actualidad, para poder trabajar a la escala comarcal ya que la delimitación actual no coincide ni con las anteriores, sean del MAPA o de otros organismos, ni con los espacios-programas utilizados anteriormente por la DGA. Se ha llevado a cabo también un trabajo de campo en varios municipios de la comarca, completando la información cuantitativa con la cualitativa mediante reuniones y entrevistas con expertos y con la población de Los Monegros. A partir de toda esta información se han elaborado las tablas y se han sacado las conclusiones pertinentes.

\section{La población en Los Monegros}

\subsection{Evolución general de la población}

A lo largo del siglo XX, la evolución poblacional de Los Monegros ha sido similar a la de otras zonas rurales de la España interior: hasta los años cuarenta, con algunas inflexiones, la población ha crecido lentamente o se ha mantenido. Es a partir de los años 60 cuando la comarca empezó a experimentar en conjunto una pérdida demográfica paulatina y constante de población, siguiendo una tendencia acorde con la dinámica de las zonas rurales españolas. La explicación de esta pérdida se encuadra dentro del marco general del éxodo rural español y aragonés (Figura 2), y las razones hay que buscarlas en el contraste

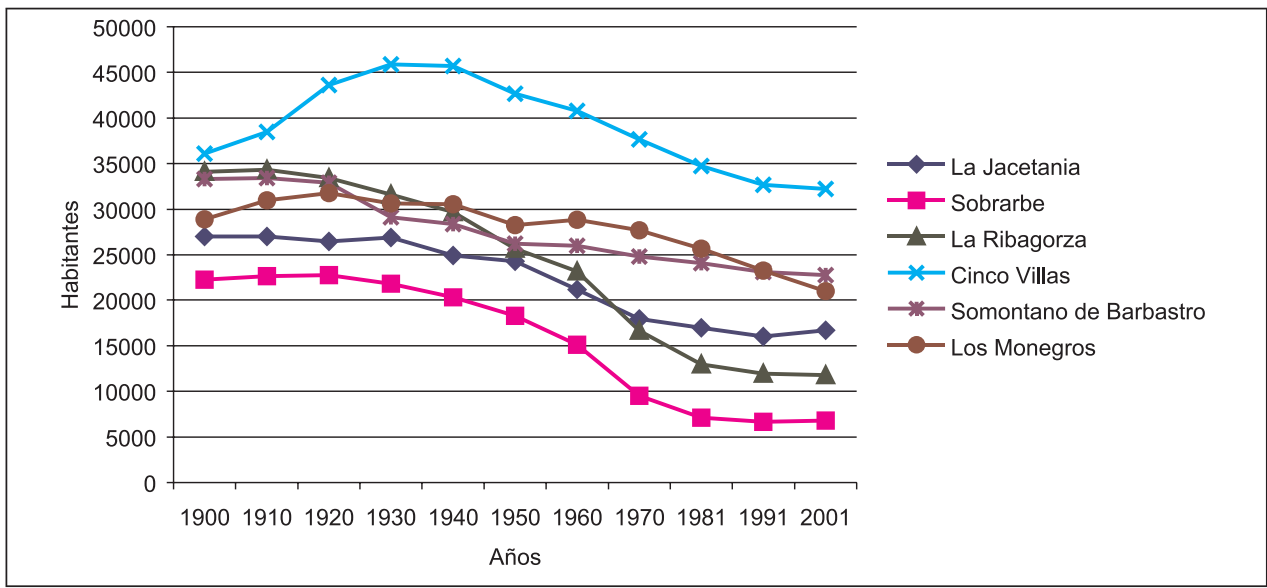

FIGURA 2. Evolución poblacional de algunas comarcas aragonesas (1900-2001).

Elaboración propia a partir de los datos del INE. 
socioeconómico existente entre la actividad económica y los recursos disponibles en dichos espacios y el de otras zonas cercanas, que resultan mucho más atractivos por las posibilidades de promoción y de bienestar que puede proporcionar, todo ello relacionado con la puesta en marcha de los Planes de Desarrollo. Se suma a este éxodo también la emigración a los países de Europa, en reconstrucción tras la guerra.

Analizando la evolución de la población monegrina, se observa que en los dos primeros decenios del siglo XX se alcanza el máximo censal, con 31.709 habitantes en el año 1920. Este aumento coyuntural respecto a las dos décadas anteriores se debe a una mejor situación relativa de las comarcas cerealistas, como la de Los Monegros, en relación con la filoxera que afecta las zonas vitivinícolas, permitiendo un aumento del terrazgo labrado en aquellas, lo que posibilitaba la retención de la población, coincidiendo con la época en que las hectáreas cultivadas aumentaron considerablemente debido a estas roturaciones, como lo explican Ferrer (1960), Bielza y Escolano (1983) y Castelló (1989).

Sin embargo, a partir de entonces las variaciones han sido negativas, con distinto ritmo según la época, iniciándose un descenso demográfico entre 1930 y 1940, año a partir del cual, como consecuencia de la de las circunstancias de postguerra, las migraciones en España se ralentizan; pero cuando en los años 50 se reanuda el proceso migratorio, la comarca de Los Monegros se verá afectada, sin que los asentamientos de colonización llevados a cabo en los nuevos regadíos en los años 60, hayan podido contrarrestar las fuertes pérdidas de los secanos.

De modo general, desde el inicio del siglo pasado hasta la actualidad la población monegrina ha descendido 7.843 habitantes, es decir, un 27,21\% respecto a 1900 , pasando de 28.815 en esta fecha a 20.972 en 2003 . No obstante, si este proceso regresivo no ha alcanzado niveles superiores, como los de otras comarcas (Tabla 1), ha sido por la atracción que ejercieron los núcleos de colonización para jóvenes activos procedentes de otros espacios como Huesca, Zaragoza, Soria, Andalucía o Extremadura.

Tabla 1

COMPARACIÓN DE LA PÉRDIDA DE POBLACIÓN ENTRE MONEGROS Y OTRAS COMARCAS ARAGONESAS SIN REGADÍO (1900-2003)

\begin{tabular}{|l|c|}
\hline Comarca & Porcentaje de pérdida de población \\
\hline Campo de Belchite & $-63,45$ \\
\hline Bajo Martín & $-35,03$ \\
\hline Maestrazgo & $-81,99$ \\
\hline Sierra de Albarracín & $-68,95$ \\
\hline Matarraña & $-63,94$ \\
\hline Monegros & $-27,21$ \\
\hline
\end{tabular}

Elaboración propia a partir de los datos del INE.

A escala municipal esta tendencia general que acabamos de explicar presenta peculiaridades en cada uno de los municipios, en los cuales este comportamiento no ha sido homogéneo, puesto que las características inherentes a los mismos, en cuanto a posibilidades de desarrollo, han variado a lo largo de los años, aunque desde 1900 hasta 1950 con mayor o menor intensidad las curvas ofrecen el mismo perfil. Desde entonces la mayoría se ha sumido en un proceso de despoblación, y muy pocos han iniciado un tímido ascenso, 
como Bujaraloz, potenciado por el tráfico de la carretera N-II, pasando de 1.057 a 1.220 habitantes entre 1960 y 1981 , con un crecimiento poblacional del orden del 15,42\%; ha crecido en mayor medida Sariñena, cuya población pasó de 3.787 a 4.455 habitantes en las mismas fechas, es decir un aumento de 17,63\%, con la instalación de nuevos poblados y cierto dinamismo por su carácter de cabecera comarcal.

\subsection{El proceso de colonización en Los Monegros}

La transformación en regadío de los secanos de Los Monegros trajo consigo un cambio de estructura de la propiedad, ya que parte de las tierras de la zona fueron adquiridas por el Instituto Nacional de Colonización (INC), asimilado a partir de 1971 por el IRYDA, y redistribuidas entre nuevos pequeños propietarios, a los cuales era necesario albergar debidamente. Siguiendo a Casas et al. (1960), el INC ha ido aplicando diferentes acciones, derivadas de las leyes de Colonización, que se han materializado en la creación de pueblos nuevos y la instalación de los respectivos colonos en las tierras expropiadas para tal finalidad. Los núcleos de colonización construidos están dotados de todos los servicios necesarios y situados de forma que los nuevos colonos tuvieran sus tierras lo suficientemente cerca para poder atenderlos tan asiduamente como fuera necesario, según la exigencia de los cultivos de regadío. Estos núcleos de colonización se recogen en la Tabla 2.

Tabla 2

LOS NÚCLEOS DE COLONIZACIÓN EN LOS MONEGROS

\begin{tabular}{|l|c|c|c|c|}
\hline Zona & Pueblo & Municipio & $\begin{array}{c}\text { Número } \\
\text { colonos }\end{array}$ & $\begin{array}{c}\text { Tamaño } \\
\text { (Ha) }\end{array}$ \\
\hline Canal Monegros & Valfonda & Torres de Barbués & 75 & 971 \\
\hline Tramo II & Frula & Almuniente & 83 & 1.200 \\
\hline Tramo II & Montesusín & Grañen & 88 & 1.710 \\
\hline Tramo II & Sodeto & Grañen & 81 & 1.565 \\
\hline Canal del Flumen & Grañen & 148 & 2.150 \\
\hline Canal del Flumen & San Lorenzo de Flumen & Grañen & 70 & 983 \\
\hline Canal del Flumen & Curbe & & & \\
\hline Canal de Monegros & \multicolumn{5}{|c|}{ Orillena } & Lanaja & 81 & 2.002 \\
\hline Tramo III & Cantalobos & Poleñino & 32 & 556 \\
\hline Tramo III & La Cartuja & Sariñena & 82 & 1.712 \\
\hline Tramo III & San Juan del Flumen & Sariñena & 68 & 2.327 \\
\hline Tramo III & \multicolumn{5}{|c|}{}
\end{tabular}

Fuente: Martínez, 1983. IRYDA.

La creación de estos diez núcleos de colonización ha contribuido al cambio de paisaje y de distribución de la población en la comarca con la implantación de los nuevos regadíos. Por otra parte, se observa que todos los pueblos no tienen el mismo tamaño. Esto ha dependido de la cantidad de tierras disponibles a su alrededor y su zona de influencia, sobre 
la que se calculó el número de colonos que podían recibir un lote y por tanto necesitaban vivienda (Tabla 2).

Los colonos que recibieron tierras fueron 808 , además de 179 vecinos de los pueblos ya existentes, antiguos propietarios de pequeñas parcelas en las zonas regables, que solicitaron un lote complementario para alcanzar el tamaño medio de explotación calculado como suficiente para el mantenimiento de una familia. En esta zona el INC adquirió un total de 18.404 Ha, parte por oferta voluntaria de sus propietarios y el resto por expropiación forzosa entre los propietarios de más de $30 \mathrm{Ha}$ de tierras incluidas en la zona regable. En un principio, con el fin de que la tierra llegara al mayor número de colonos posibles se repartieron lotes de unas $10 \mathrm{Ha}$ e incluso menores (8Ha), aunque siempre en tierras de calidad inferior se acrecentaba la extensión del lote; más tarde los lotes adjudicados han sido mayores, llegando hasta 12 Ha, siendo el criterio económico, según Castelló (1989), alcanzar iguales ingresos por unidad de trabajo que los de un obrero industrial especializado.

\subsection{Evolución de la población en los municipios de regadío}

La instalación de nuevos pobladores sin duda afectó al contingente demográfico en ese momento. Pero las teorías sobre las transformaciones agrarias hacían suponer que esta tendencia se mantendría y que frenaría el éxodo rural, fijando la población, al crear más riqueza y con ella más puestos de trabajo en agricultura, industria y servicios. Sin embargo, solo temporalmente y en los municipios afectados por la implantación de los núcleos de colonización, se aprecia un incremento en el número de habitantes. En la Tabla 3, puede contrastarse la evolución de municipios de secano y de regadío.

Tabla 3

EVOLUCIÓN POBLACIONAL EN UNOS MUNICIPIOS DE SECANO Y REGADÍO EN LOS MONEGROS

\begin{tabular}{|c|c|c|c|c|c|c|c|c|c|c|c|c|c|c|c|}
\hline & Municipios & 1900 & 1910 & 1920 & 1930 & 1940 & 1950 & 1960 & 1970 & 1981 & 1986 & 1991 & 1996 & 1999 & 2003 \\
\hline \multirow{6}{*}{$\begin{array}{l}\text { Municipios } \\
\text { de Regadío }\end{array}$} & $\begin{array}{c}\text { Alberuela de } \\
\text { Tubo }\end{array}$ & 238 & 219 & 244 & 232 & 249 & 262 & 267 & 184 & 465 & 469 & 439 & 404 & 388 & 361 \\
\hline & Grañen & 1431 & 1478 & 1460 & 1310 & 1363 & 1670 & 2622 & 3599 & 2482 & 2408 & 2162 & 2062 & 2006 & 2009 \\
\hline & Lalueza & 867 & 940 & 1020 & 1091 & 1151 & 1082 & 1217 & 1056 & 1558 & 1469 & 1389 & 1292 & 1262 & 1152 \\
\hline & Lanaja & 1839 & 2090 & 2088 & 2103 & 2255 & 1980 & 2159 & 2017 & 1963 & 1941 & 1771 & 1651 & 1574 & 1496 \\
\hline & Sariñena & 3963 & 4354 & 4446 & 3917 & 4392 & 3787 & 3993 & 4202 & 4338 & 4455 & 4227 & 4058 & 4026 & 3987 \\
\hline & $\begin{array}{l}\text { Torres de } \\
\text { Barbués }\end{array}$ & 202 & 238 & 250 & 228 & 233 & 200 & 189 & 544 & 492 & 467 & 428 & 370 & 371 & 336 \\
\hline \multirow{6}{*}{$\begin{array}{c}\text { Municipios } \\
\text { de Secano }\end{array}$} & Bujaraloz & 1510 & 1542 & 1481 & 1303 & 1391 & 1137 & 1057 & 1148 & 1220 & 1147 & 1074 & 1035 & 1018 & 1006 \\
\hline & Leciñena & 1576 & 1798 & 2107 & 2152 & 1952 & 1993 & 1730 & 1554 & 1542 & 1534 & 1476 & 1413 & 1378 & 1296 \\
\hline & Monegrillo & 824 & 902 & 853 & 718 & 827 & 798 & 689 & 702 & 624 & 594 & 567 & 553 & 538 & 517 \\
\hline & Perdiguera & 725 & 807 & 874 & 796 & 726 & 783 & 680 & 595 & 548 & 536 & 480 & 447 & 446 & 569 \\
\hline & Farlete & 524 & 559 & 528 & 548 & 610 & 637 & 587 & 565 & 541 & 494 & 496 & 468 & 457 & 451 \\
\hline & La Almolda & 1246 & 1253 & 1273 & 1210 & 1256 & 1098 & 991 & 880 & 826 & 782 & 764 & 717 & 701 & 653 \\
\hline
\end{tabular}

Fuente: Censo de Población y Padrón Municipal de habitantes, INE. Elaboración propia. 
Analizando la evolución de la población en los municipios de secano y los del regadío en la comarca de Los Monegros, se pone de manifiesto que el comportamiento no ha sido homogéneo en todos ellos. En efecto, mientras que los de secano padecían un despoblamiento constante iniciado en los años 50, los de regadío, tras un incremento de sus respectivas poblaciones, generado por los procesos de atracción de población que ejercieron los núcleos de colonización en Grañén, Lanaja, Sariñena y Lalueza tras la puesta en regadío con el Plan de Riegos del Alto Aragón, han experimentado un descenso poblacional a partir de los años 70, que afectó también a la mayoría de sus núcleos de colonización, pasando de 3.843 en dicha fecha a tan sólo 2.826 habitantes actualmente, con una perdida del $26,46 \%$ en este periodo, ya que únicamente La Cartuja de Monegros y San Juan de Flumen han conseguido mantener su población en estos treinta últimos años, tal y como puede verse en la Tabla 4.

Tabla 4

EVOLUCIÓN DE LA POBLACIÓN EN LOS NÚCLEOS DE COLONIZACIÓN (1970-2005)

\begin{tabular}{|c|c|c|c|c|}
\hline Municipios de ubicación & Núcleos & 1970 & 1999 & 2005 \\
\hline Alberuela de Tubo & Sodeto & 351 & 242 & 238 \\
\hline Almuniente & Frula & 502 & 394 & 362 \\
\hline Grañen & $\begin{array}{l}\text { Curbe } \\
\text { Montesusín }\end{array}$ & $\begin{array}{l}374 \\
402\end{array}$ & $\begin{array}{l}265 \\
298\end{array}$ & $\begin{array}{l}238 \\
284\end{array}$ \\
\hline Lanaja & $\begin{array}{l}\text { Cantalobos } \\
\text { Orillena }\end{array}$ & $\begin{array}{l}196 \\
410\end{array}$ & $\begin{array}{l}114 \\
291\end{array}$ & $\begin{array}{l}102 \\
272\end{array}$ \\
\hline Lalueza & San Lorenzo de Flúmen & 599 & 484 & 406 \\
\hline Sariñena & $\begin{array}{l}\text { La Cartuja de Monegros } \\
\text { San Juan de Flúmen }\end{array}$ & $\begin{array}{l}322 \\
269\end{array}$ & $\begin{array}{l}327 \\
366\end{array}$ & $\begin{array}{l}308 \\
368\end{array}$ \\
\hline Torres de Barbués & Valfonda de Santa Ana & 418 & 282 & 248 \\
\hline Total población & & 3843 & 3063 & 2826 \\
\hline
\end{tabular}

Fuente: Nomenclator y Padrón Municipal de habitantes, INE. Elaboración propia.

Esto demuestra que la puesta en regadío en Los Monegros no ha tenido una clara repercusión en el aumento de la población y que los incrementos demográficos de algunos municipios con núcleos de colonización se deben a la desaparición administrativa, entre 1970 y 1981, de otros como los de Lastanosa, Pallaruelo de Monegros y Marcén, que quedan anexionados, los dos primeros a Sariñena y el tercero a Lalueza. No obstante, en algunos casos concretos, como Lanaja, estas entidades poseen tan poca población que no logran compensar las pérdidas, y entonces al descenso demográfico del municipio principal se añade el correspondiente de los núcleos anexionados, perdiendo cantidades cercanas al $10 \%$ de su población, ya que el municipio ha experimentado un descenso demográfico claro entre 1960 y 1981, pasando de 2.159 habitantes a 1.963. Se confirma así, tal y como lo planteaba Castelló (1989), que en las primeras décadas después de la puesta en regadío la población no se había mantenido ni siquiera en todos los municipios con núcleos de colonización. A partir de esta fecha y hasta el momento actual, la dinámica no ha cambiado, tal como se aprecia en la Tabla 4. 
Cabe señalar además, que la densidad demográfica de Los Monegros, con tan solo 7,58 habitantes $/ \mathrm{Km}^{2}$, es inferior a la de otra zona similar de regadío como es la comarca de Cinco Villas, que cuenta con 10,6 habitantes $/ \mathrm{Km}^{2}$ y de la provincia de Huesca, con 13,5 habitantes $/ \mathrm{Km}^{2}$, siendo estas ya de por sí muy bajas. No obstante, en la zona con riego se alcanzan los 15,86 habitantes/ $\mathrm{Km}^{2}$, aunque ciertamente, se pone de relieve que la repercusión del regadío en este aspecto no ha sido muy espectacular.

\subsection{Otros aspectos de la población}

Uno de los problemas de la comarca monegrina es el saldo migratorio negativo, con un éxodo que se ha dirigido tradicionalmente hacia ciudades como Zaragoza, Barcelona o Huesca. No obstante, la intensidad de esta emigración es menor a la de otras comarcas aragonesas, dado que los núcleos de colonización no sólo han amortiguado el abandono de población de los pueblos durante los años de su implantación, sino que se convirtieron, durante algún tiempo, en lugares de atracción para la población activa de otros municipios de la comarca o de la provincia y de otras comunidades autónomas, como ya se ha dicho.

El resultado de los movimientos naturales y migratorios queda patente en la estructura biológica de la población, que nos permite conocer la distribución de sus habitantes por edad y por sexo (Figura 3).

Tabla 5

INDICADORES DE ESTRUCTURA DEMOGRÁFICA

\begin{tabular}{|l|c|c|}
\hline Indicadores de estructura & Monegros & Aragón \\
\hline Edad media & 48,06 & 42,88 \\
\hline Índice de juventud & 0,43 & 0,83 \\
\hline Índice de vejez & 2,57 & 1,20 \\
\hline Tasa de Envejecimiento & 30,28 & 21,48 \\
\hline
\end{tabular}

Fuente: IAE, 2001. Elaboración propia.

Examinando los indicadores de estructura demográfica (Tabla 5), se observa un fuerte envejecimiento, ya que el 30,28\% tiene más de 64 años y la edad media de la población monegrina es de 48,06 años, que es bastante superior a la de Aragón (42,88 años), ya de por sí bastante elevada. Además, el índice de juventud de la comarca de Monegros es muy bajo con 0,43, y claramente inferior al de Aragón, que es del orden de 0,83, observación válida para todos los índices de juventud de los municipios monegrinos que están muy por debajo de los aragoneses. Esos índices de envejecimientos superiores a los regionales, influyen negativamente en cantidad de población activa, lo que hace difícil llevar a cabo cualquier tipo de iniciativa para reanimar la zona.

En cuanto a la distribución por sexo, hay un ligero predominio de los varones que suman el 51,4\% de la población con 10.785 hombres. En cambio, las mujeres son 10.204 y representan tan sólo el $48,61 \%$. El hecho que la proporción de los hombres supere a la de las mujeres y que, por tanto, el índice de masculinidad supere a 100, es un síntoma de debilidad demográfica y desequilibrio en la comarca, a causa de sobre todo la emigración, que ha afectado y en mayor número a las mujeres del medio rural. Este desajuste en Los Monegros plantea problemas sociodemográficos, ya que incide en un menor índice de nup- 


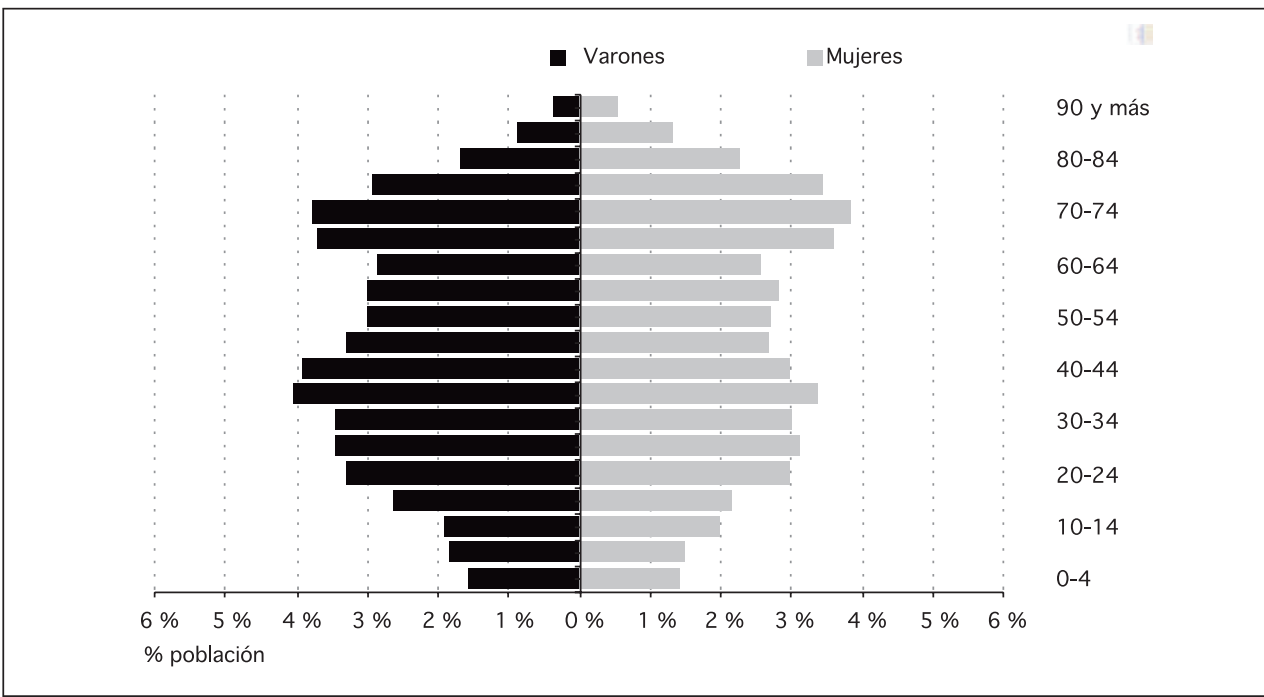

Figura 3. Pirámide poblacional de Los Monegros en 2001.

Fuente: IAE.

Sariñena

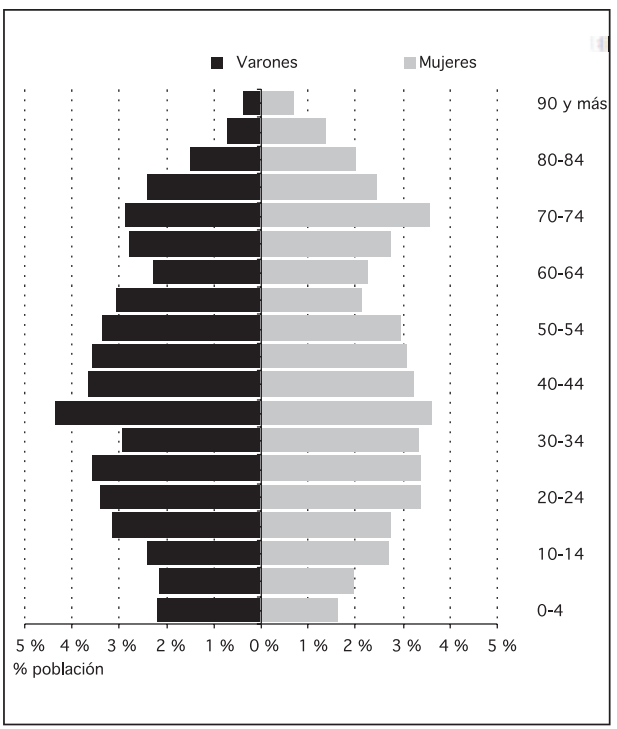

Monegrillo

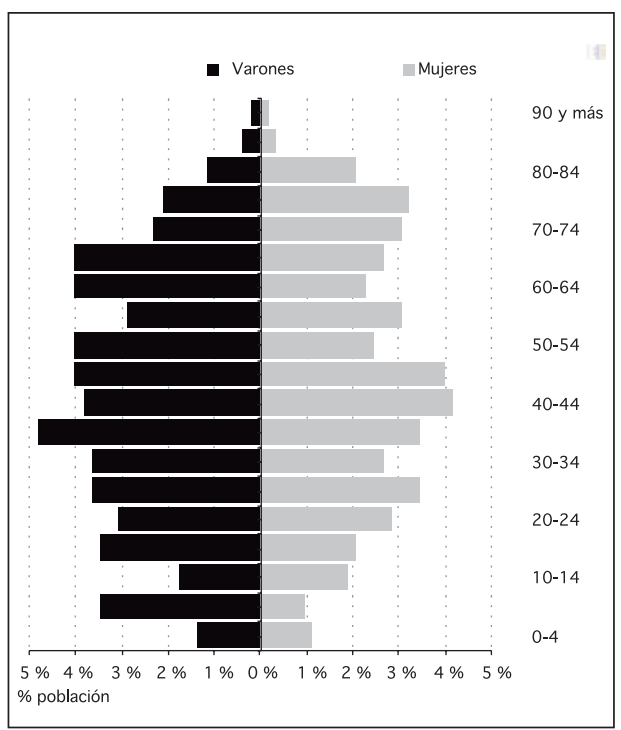

Figura 4. Comparación de las pirámides poblacionales de Sariñena y Monegrillo del año 2001. Fuente: $I A E$. 
cialidad y por tanto, en un menor número de hijos. Que haya también un alto porcentaje de solteros (no voluntarios) plantea problemas de estructura social.

Estos problemas quedan reflejados en la pirámide poblacional (Figura 3) que se estrecha en la base y se ensancha en la parte superior, lo que indica un fuerte envejecimiento de la población, común no sólo a los municipios de la comarca (ya sea de regadío o de secano), como lo pone en evidencia la Figura 4; sino también a todas las zonas rurales aragonesas. Sin embargo, pese a que en todos los municipios la estructura por edad y sexo es muy desequilibrada, las pirámides que ilustran la Figura 4 permiten apreciar que la ubicación del regadío y el tamaño demográfico influyen en una estructura algo mejor.

Esta análisis nos demuestra que la población monegrina está en declive y claramente envejecida, lo que ha influido de una manera negativa en la evolución del crecimiento vegetativo comarcal, puesto que el continuado descenso de la población y el deterioro de su estructura ha dado lugar a unas pautas en la natalidad y mortalidad que se repiten en casi todo el espacio aragonés, ya que la natalidad ha experimentado un descenso importante en la mayor parte de los municipios monegrinos.

En efecto, analizando la Tabla 6, puede verse que el número de nacimientos ha descendido un $28 \%$ del 1991 al 2001, con una tasa de natalidad de $4.9 \%$ en el año 2001, muy por debajo de la media aragonesa que es $8,6 \%$. En cuanto a la mortalidad, en cifras absolutas ha variado poco y ha pasado de 245 defunciones en el año 1991 a 255 diez años más tarde, aunque en los años 1996 y 1999 han alcanzado los valores máximos con 287 y 281 fallecimientos respectivamente. En el año 2001, la tasa de mortalidad es de $12 \%$, lo que ha repercutido negativamente, como se ha dicho, en el crecimiento vegetativo, que se sitúa en $-7,1 \%$ o, con peores perspectivas todavía que la media de Aragón, cuyo crecimiento vegetativo es de $-2 \%$ en 2001. En suma, Los Monegros han perdido en el último periodo intercensal en su dinámica natural, 151 habitantes.

Tabla 6

EVOLUCIÓN DEL MOVIMIENTO NATURAL DE LA POBLACIÓN (1991-2001)

\begin{tabular}{|c|c|c|c|c|c|c|}
\hline \multirow{2}{*}{} & \multicolumn{2}{|c|}{ Mortalidad } & \multicolumn{2}{c|}{ Natalidad } & \multicolumn{2}{c|}{ Crecimiento vegetativo } \\
\cline { 2 - 7 } & Número & $\%$ Nómero & $\%$ Número & $\%$ \% \\
\hline 1991 & 245 & 10,6 & 145 & 6,3 & -100 & $-4,3$ \\
\hline 1992 & 263 & 11,4 & 138 & 6,0 & -125 & $-5,4$ \\
\hline 1993 & 230 & 10,1 & 112 & 4,9 & -118 & $-5,2$ \\
\hline 1994 & 244 & 10,8 & 140 & 6,2 & -104 & $-4,6$ \\
\hline 1995 & 277 & 12,4 & 137 & 6,1 & -140 & $-6,3$ \\
\hline 1996 & 287 & 13,1 & 109 & 5,0 & -178 & $-8,1$ \\
\hline 1997 & 241 & 11,0 & 129 & 5,9 & -112 & $-5,1$ \\
\hline 1998 & 278 & 12,9 & 110 & 5,1 & -168 & $-7,8$ \\
\hline 1999 & 281 & 13,1 & 115 & 5,4 & -166 & $-7,7$ \\
\hline 2000 & 247 & 11,6 & 114 & 5,4 & -133 & $-6,2$ \\
\hline 2001 & 255 & 12,0 & 104 & 4,9 & -151 & $-7,1$ \\
\hline
\end{tabular}

Fuente: IAE. Elaboración propia. 


\subsection{El descenso de la población activa agrícola}

Este declive demográfico ha influido negativamente en la población activa del sector agrario. En efecto, se advierte que la agricultura ha dejado de ser el principal proveedor de empleo en la comarca, pasando de 36\% del total de la población ocupada en el año 1991, a tan sólo el $27 \%$ en el año 2002 (Tabla 7). No obstante, este porcentaje es claramente superior al aragonés, que apenas alcanza el $6 \%$, lo que pone de manifiesto la importancia de este sector de actividad en Los Monegros, que sin duda fue impulsado por las transformaciones del Plan de Riegos del Alto Aragón.

Tabla 7

EVOLUCIÓN DE LA POBLACIÓN OCUPADA POR SECTOR DE ACTIVIDAD (1981-2002) EN \%

\begin{tabular}{|l|c|c|c|}
\hline Sectores & $\mathbf{1 9 8 1}$ & $\mathbf{1 9 9 1}$ & $\mathbf{2 0 0 2}$ \\
\hline Agricultura & 52 & 36 & 27 \\
\hline Industria & 8 & 15 & 12 \\
\hline Construcción & 14 & 14 & 19 \\
\hline Servicios & 26 & 35 & 42 \\
\hline
\end{tabular}

Fuente: Renta municipal de Aragón, IAE y INAEM. Elaboración propia.

Todavía en 1981 la población agraria suponía el 52\% de los activos. Esta alta cifra está relacionada con la propia estructura productiva de la comarca, con una fuerte base agraria que consolidó el regadío y muy pocas ofertas de otro tipo de trabajo. Pero también incidía la crisis industrial general de este periodo. Sin embargo, a partir de estas fechas la población dedicada al sector agrario ha descendido por varias causas: el abandono de parcelas marginales de escaso rendimiento y la consiguiente merma de las tierras, con cultivos extensivos o semi-extensivos. Incluso parte de los agricultores trabajan la tierra a tiempo parcial. Tampoco son ajenas a este proceso las repercusiones de la PAC, propiciando el abandono de tierras y la jubilación anticipada, el propio envejecimiento de la población y la dificultad de los jóvenes para adquirir tierras que les permitan tener su propia explotación. En este caldo de cultivo, la emigración ha afectado especialmente a este segmento de la población activa.

La actividad de la población monegrina siempre ha estado condicionada por la infraestructura productiva de cada municipio y por la pirámide de edades. A pesar de que el sector agrario ha sido determinante de la economía de este espacio territorial, en las dos últimas décadas se ha ido produciendo un incremento de actividades no agrarias, que han generado empleo para la población activa más joven y con mayor nivel de cualificación profesional, aunque solo los servicios superan al sector agrario.

\section{Las causas de esta situación}

El análisis que acaba de presentarse deja patente que el objetivo teórico de fijar o incrementar población por la transformación en regadío no se ha cumplido en esta comarca. Las causas son múltiples: en primer lugar tienen que ver con el propio proceso de colonización, en el que se optó por adjudicar lotes de pequeña dimensión, en función del sistema agrario del momento; como segundo factor que ha incidido en la dinámica demográfica 
puede señalarse la elección de cultivos por parte de los colonos; junto a esto, la creciente mecanización, que no retiene mano de obra; finalmente, la escasa o nula integración vertical de la agricultura con la ganadería y la agroindustria. Habría que añadir un último factor: la proximidad de Zaragoza y Huesca que propicia el cambio de residencia por el exceso número de jornadas de trabajo que requiere el tipo de cultivos.

\subsection{El tamaño de las explotaciones}

Tras la puesta en marcha del regadío en la comarca ha habido un proceso de transformación, generando un cambio en la distribución de la superficie cultivada, la propiedad, la parcelación y la explotación. Entre ellos, el más destacado es la disminución de la población agraria, y como consecuencia, el descenso del número de explotaciones y, por lógica, el incremento del tamaño medio, pasando de 47 Ha en 1972 a 61 Ha en 1999. Esto ha dado origen a un proceso generalizado de absorción de las pequeñas explotaciones por agricultores-empresarios de las medianas, en busca de la rentabilidad deseada. Sin embargo, a pesar de del incremento general del tamaño medio de las explotaciones, hay una neta fragmentación de las de regadío, donde dominan las de 10-20 Ha, puesto que los lotes repartidos en el proceso de colonización tienen estas dimensiones. A modo de ilustración, en la Figura 5 puede apreciarse bien la diferente distribución de las explotaciones según el tamaño en un municipio de secano, Farlete, y otro de regadío, Grañen.

Llama la atención la alta proporción de las explotación de 0-5 Ha, lo que refleja el mantenimiento del minifundio. Esos reducidos tamaños de las explotaciones en regadío representan un gran handicap para lograr la rentabilidad de parte de los agricultores monegrinos, como ya advirtió Castelló (1989). Este tamaño solo sería suficiente para competir en el mercado actual con un uso muy intensivo y cultivos muy rentables.

\subsection{El carácter extensivo (o semi-extensivo) de los cultivos}

Sin duda, la transformación en regadío ha causado un cambio importante en los cultivos y la producción agrícola de la comarca, ya que el cultivo más extendido era el trigo, al que

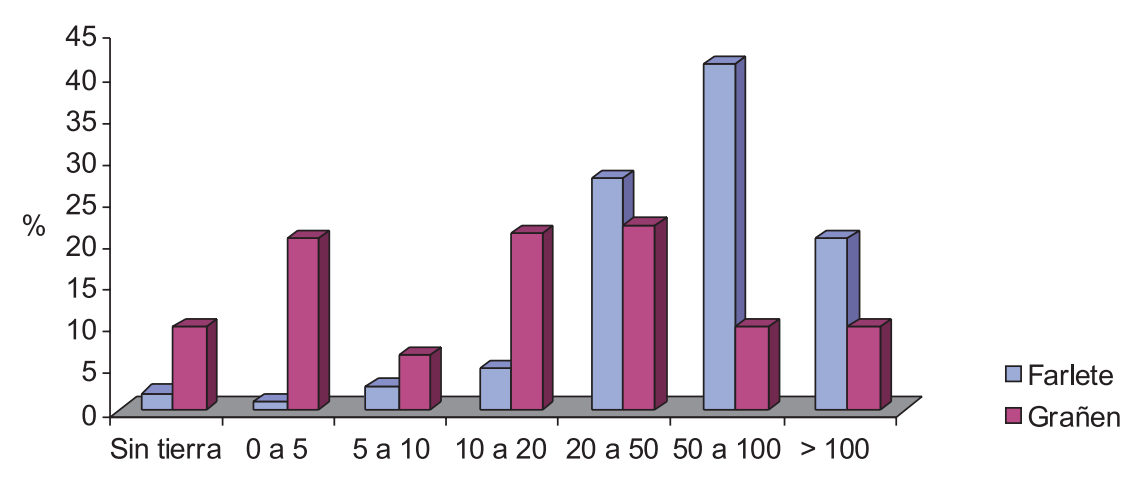

$\mathrm{Ha}$

FiguRA 5. Comparación del tamaño medio de las explotaciones (Farlete-Grañen).

Fuente: Elaboración propia a partir de los datos de los Censos Agrarios. 


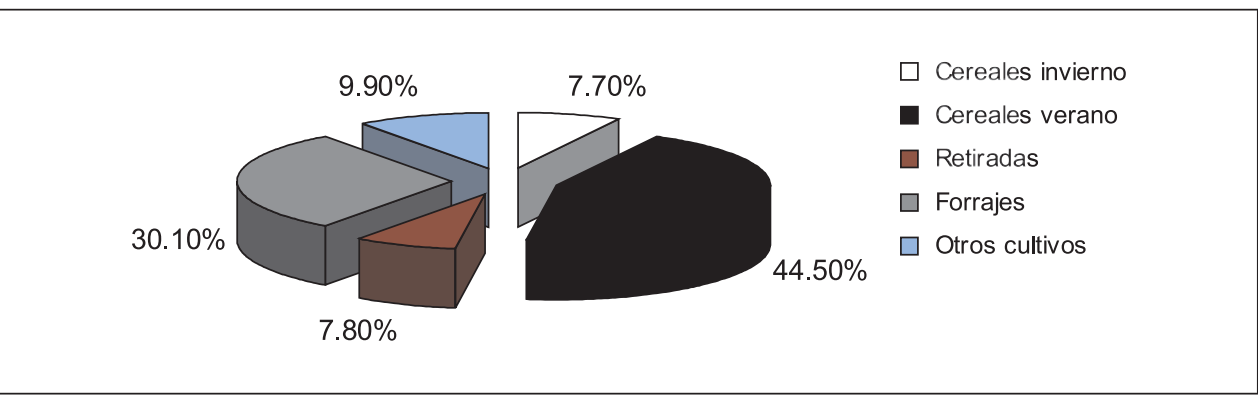

Figura 6. Los cultivos más relevantes en el regadío en Los Monegros, 2001.

Fuente: 1T del 2001 de la DGA. Elaboración propia.

le seguían otros cereales de invierno, como la cebada, el centeno y la avena; alguna viña, más unos pocos olivos y almendros completaban la gama a la que dedicaban sus tierras los agricultores monegrinos. Actualmente, en el conjunto de la comarca los cereales de invierno siguen manteniendo un puesto principal en cuanto a extensión, con alguna modificación, como es que ahora se siembre más cebada que trigo, por su mayor rendimiento y por la política llevada a cabo desde los años 70 y se siguen sembrando avena y centeno, aunque en escasa proporción.

Pero si se analiza el regadío (figura 6), puede verse que aunque los cereales representan también el principal tipo de cultivo herbáceo, con 52\% del total, aunque a los cereales de invierno se han sumado los cultivos verdes o de primavera y verano. Entre ellos, el maíz ocupa la mayor extensión en las tierras regadas, con $27.037 \mathrm{Ha}$, lo que supone el 37,25\%. En los últimos años, el arroz se ha ido extendiendo a partir de la entrada en funcionamiento de la zona regable del Canal de Monegros, ocupando una superficie de 5.024 Ha, especialmente en el fondo de los valles, ya que se ha introducido como solución al importante problema que presenta la excesiva salinidad de los suelos en gran parte del territorio monegrino. El arroz y el maíz, que son cereales de verano, ocupan el $44 \%$ de la superficie de herbáceos en el regadío. En cuanto a la cebada y el trigo, que son cereales de invierno, ocupan unas extensiones inferiores con $7,7 \%$ del total de los herbáceos en el regadío. Los cultivos forrajeros, y entre ellos, la alfalfa ocupan más del $30 \%$ de la superficie del regadío, con una extensión de 19.794 Ha; en cuanto a los cultivos industriales, básicamente el girasol, cuentan con tan solo el $8,8 \%$.

Tanto los cultivos hortícolas como los frutales tienen una reducida incidencia dentro de la actividad agraria monegrina, y su presencia es meramente testimonial, con una superficie de 213 Ha y 513 Ha respectivamente. Ciertamente el clima y la calidad de los suelos no son demasiado adecuados para este tipo de cultivos; pero también esta escasez es debida a la falta de costumbre y de tradición, a lo que no ha ayudado la PAC, que no los subvenciona, la ausencia de cooperativas que se encarguen de la comercialización de los productos o la implantación de invernaderos o de cultivos bajo plástico.

En suma, hay que destacar el carácter extensivo o semi-extensivo de los cultivos del regadío monegrino que no necesitan una dedicación total, lo que ha incentivado la existencia de la agricultura a tiempo parcial, y ha provocado una descenso en la población activa que se dedica a la agricultura, tanto por la poca necesidad de mano de obra como por el tipo de cultivos y el reducido tamaño de las explotaciones, que, como en otras zonas en las que la agricultura a tiempo parcial se ha incrementado, ha hecho necesario según Etxezarreta (1985) compartir el trabajo agrario. 


\subsection{La mecanización de las explotaciones agrarias}

El parque de maquinaria agraria en Los Monegros se ha incrementado, alcanzando unos niveles más que aceptables de mecanización, que es incluso excesiva en algunos municipios, teniendo en cuenta el coste económico de la misma en relación con la superficie labrada y el tamaño de las explotaciones, tal y como se puede apreciar en la Tabla 8, en la que se presenta la evolución del censo de maquinaria agrícola existente en las explotaciones entre 1982 y 1999.

Tabla 8

EVOLUCIÓN DE LA MECANIZACIÓN DE LAS EXPLOTACIONES EN MONEGROS (1982-1999)

\begin{tabular}{|l|c|c|c|c|c|}
\hline Años & Total máquinas & Tractores & Motocultores & Cosechadoras & Otras máquinas \\
\hline 1982 & 4.685 & 2.968 & 389 & 863 & 465 \\
\hline 1989 & 4.704 & 3.095 & 504 & 416 & 689 \\
\hline 1999 & 3.973 & 3.003 & 620 & 297 & 53 \\
\hline
\end{tabular}

Fuente: Censos Agrarios de 1982, 1989 y 1999. Elaboración propia.

Se observa un crecimiento a lo largo de la década de los ochenta y un descenso considerable en la siguiente. Sin embargo, pese a este descenso el grado de mecanización de la comarca sigue siendo alto, si tenemos en cuenta el carácter semi-extensivo o extensivo de las explotaciones en el regadío, lo que impide traer más mano de obra para trabajar en el campo monegrino.

Hay que subrayar, también, que la concentración de las labores agrarias en periodos cortos, gracias a esta mecanización, ha permitido que una parte de los agricultores residan en ciudades como Huesca o Zaragoza.

\subsection{Desconexión del regadío con la ganadería y la agroindustria}

En lo que respecta a la integración con la ganadería, la conexión se limita a una primera elaboración de piensos para el ganado lanar o bovino, mientras que la cría del ganado porcino, que proporciona actualmente la mayor parte de la renta ganadera de la comarca, es completamente independiente de los cultivos, evitando así que estos últimos, y sobre todo los de regadío, incrementasen su valor añadido y, por lo tanto, la renta comarcal y los puestos de trabajo a través de su capacidad de arrastre. En cuanto a la industria agroalimentaria ligada al regadío es escasa, con tan solo 40 empresas repartidas en la totalidad de la comarca, ofreciendo 258 puestos de trabajo, limitándose principalmente a las deshidratadoras y el secado, y la mayoría se encuentran en los municipios del regadío (Tardienta, Sariñena, Grañen, Lanaja, y Lalueza), tal y como muestra la Tabla 9.

Las agroindustrias de productos hortofrutícolas brillan por su ausencia, por las negativas experiencias que ha habido con algunas empresas foráneas, como la «Gran Conservera de Sariñena», entre los años 60 y 80 y el fracaso de algunas iniciativas posteriores como la de la «Cooperativa Hortícola Flumen-Monegros» a mediados de los años 80. Las dificultades atravesadas por el cooperativismo en el sector agrícola, que se refleja en la ausencia o/y la escasez de las cooperativas, provocan un problema de comercialización de productos, a lo que se suma la escasa iniciativa particular, la deficiente formación empresarial del 
Tabla 9

LAS AGROINDUSTRIAS SEGÚN EL NÚMERO, NÚMERO DE TRABAJADORES Y LOS MUNICIPIOS

\begin{tabular}{|c|c|c|c|}
\hline Tipo de agroindustria & Número & $\begin{array}{l}\text { Número de } \\
\text { trabajadores }\end{array}$ & Municipios \\
\hline $\begin{array}{l}\text { Deshidratación y manipulación } \\
\text { de materias primas para forraje } \\
\text { (alfalfa, pelet granulado, piensos, } \\
\text { maíz, etc) }\end{array}$ & 6 & 83 & $\begin{array}{l}\text { Bujaraloz, Sariñena, Grañen, } \\
\text { Lalueza, Lanaja. }\end{array}$ \\
\hline $\begin{array}{l}\text { Elaboración de productos cárni- } \\
\text { cos (Carnes, fabricación de em- } \\
\text { butidos y fiambres: Salchichas, } \\
\text { Longaniza, chorizo, Panceta, pa- } \\
\text { tés, etc) }\end{array}$ & 6 & 24 & $\begin{array}{l}\text { Bujaraloz, Tardienta, Grañen, } \\
\text { Sariñena, Alcubierre. }\end{array}$ \\
\hline $\begin{array}{l}\text { Panadería (Pan, tortas, bollería, } \\
\text { confitería, pastelería, magdalenas, } \\
\text { bizcochos, repostería, etc) }\end{array}$ & 17 & 47 & $\begin{array}{l}\text { Bujaraloz, Tardienta, Grañen, } \\
\text { Sariñena, Lalueza, Lanaja, } \\
\text { Almuniente, Peñalba, } \\
\text { Albalatillo, Sena, Villanueva } \\
\text { de Sigena, Sangarrén. }\end{array}$ \\
\hline $\begin{array}{l}\text { Secado y manipulación de cerea- } \\
\text { les (Arroz, etc) }\end{array}$ & 4 & 6 & $\begin{array}{l}\text { Grañen, Lanaja, Capdesaso, } \\
\text { Robres. }\end{array}$ \\
\hline Fabricación de gaseosa y sifones & 1 & 6 & Sariñena. \\
\hline $\begin{array}{l}\text { Elaboración de harina y subpro- } \\
\text { ductos de trigo }\end{array}$ & 2 & 68 & Sariñena. Tardienta. \\
\hline $\begin{array}{l}\text { Manipulación de productos horto- } \\
\text { frutícolas }\end{array}$ & 1 & 12 & Sariñena. \\
\hline $\begin{array}{l}\text { Reparación de herramientas y ma- } \\
\text { quinaria agrícola y forja }\end{array}$ & 2 & 8 & Sariñena, Peñalba. \\
\hline Producción de aceite de oliva & 1 & 4 & Alberuela de Tubo. \\
\hline Total empresas agroalimentarias & 40 & 258 & \\
\hline
\end{tabular}

Fuente: DGA, 2005. Elaboración propia.

productor agrícola y el bajo índice de especialización industrial $(0,55)$, que son las otras causas fundamentales del retraso y de la escasez de este tipo de industrias, a pesar de la constatación de su interés. 


\section{Conclusiones y consideraciones finales}

Del análisis llevado a cabo en este estudio para ver la incidencia de la transformación agraria que supone el regadío en la sociedad de la comarca de Los Monegros, puede concluirse que la evolución de la población, su estructura y su actividad ponen de relieve que hasta la actualidad, la puesta en marcha de los nuevos regadíos no ha supuesto el freno total a la emigración y el proceso regresivo, iniciado hace años en la comarca, continúa, aunque esta pérdida de población sea menor en los municipios de regadío, donde el proceso se ha ralentizado gracias a los nuevos pobladores de los núcleos de colonización, mayoritariamente jóvenes activos. Este fracaso de uno de los objetivos de la transformación en regadío puede explicarse al no haberse producido un gran cambio en los cultivos, manteniéndose de forma general el monocultivo cerealista, con carácter extensivo o semi-extensivo, en unas explotaciones con un reducido tamaño y con elevados índices de mecanización.

La edad y la formación de los agricultores, y sobre todo, la falta o la escasez de una integración vertical con la agroindustria frenan la posibilidad de obtener mayores rendimientos y no facilitan la absorción de mano de obra, e impiden que se introduzca cultivos de primor y se propicie una base de desarrollo sostenible y explican porqué las reformas agrarias que sucedieron en Los Monegros no han logrado su objetivo principal, que era fijar la población y, frenar la emigración, suponiendo un gran handicap para el desarrollo de la zona, siendo la causa de su estancamiento.

Las posibilidades económicas de una comarca tan escasamente poblada como Los Monegros dependen de la capacidad de retención de la población activa y de la atracción que propicie la venida de nuevos pobladores. Es básica la especialización económica del territorio: formación, capacidad de emprender, capacidad de asociarse, y tener las posibilidades económicas que permitan materializar las iniciativas propuestas. El regadío puede propiciar esta especialización con una adecuada planificación e integración vertical con las agroindustrias, que al fomentarla podrían ser un verdadero motor de desarrollo a través de su capacidad de arrastre, favoreciendo el asentamiento de población joven y de edad intermedia, disminuyendo la dependencia hacia otras zonas y provocando un cambio desde posiciones individualistas hacia otras más cooperativistas y empresariales, ya que la industria agroalimentaria ligada al regadío es escasísima, limitándose a deshidratadoras y poco más.

\section{Bibliografía y fuentes}

BIELZA, V., ESCOLANO, S. (1983): «Los Monegros». En Geografía de Aragón. Tomo 4, pp. 75113. Guara Editorial. Zaragoza.

BIELZA, V. (1987): Geografía Humana de Aragón. Oikos-Tau Ediciones. Barcelona.

CAI. (2005): El anuario de empleo y población en los municipios y comarcas de Aragón 2003. Ministerio de Trabajo y Asuntos Sociales. Zaragoza.

CAI. (2005): El mercado laboral en las comarcas aragonesas: balance anual 2004. Gobierno de Aragón. Zaragoza.

CAMARERO, L.A. (1992): El mundo rural español en la década de los noventa: ¿renacimiento o reconversión? Documento Social $\mathrm{N}^{\circ} 87$.

CASAS, J.M. et al. (1960): Aragón: 4 Ensayos, ver pp. 152-178. Banco de Aragón. Zaragoza.

CASTELLÓ, A. (1989): Propiedad, uso y explotación de la tierra en la comarca oscence. Instituto de Estudios Altoaragoneses. Huesca.

DGA. (2003): Datos Agrarios Básicos en Las comarcas y Delimitaciones Comarcales de Aragón 2001-2002. Departamento de Agricultura. Gobierno de Aragón. Zaragoza. 
DGA. (2005): Censo de las empresas industriales en Aragón. Gobierno de Aragón. Zaragoza.

ETXEZARRETA (1985): La agricultura insuficiente, Instituto de Estudios Agrarios, Pesqueros y Alimentarios, MAPA. Madrid.

FAUS, M.C., HIGUERAS, A. (1986): «Rural depopulation», en Regional Conference on Mediterranean Countries. Sessions of Commission of Population Geography. Departamento de Geografía y Ordenación del Territorio. Universidad de Zaragoza.

FAUS, M.C., HIGUERAS, A. (2000): «Does a demographic deficit exist?». Applied Geography 20, pp. 243-253.

FERRER, M. (1960): «La personalidad geográfica de Monegros», en Geographica. Año VII, pp. 59-88. Departamento de Geografía Aplicada. Instituto Elcano. CSIC. Madrid.

FRUTOS, L.M. (1994): «Población y mercado de trabajo». Economía de las Comunidades Autónomas: El Valle Medio del Ebro, pp. 47-70. Ibercaja. Zaragoza.

FRUTOS, L.M. (1998): «Los espacios en declive demográfico: problemas y posibilidades de recuperación». Economía Aragonesa, nº 2, pp. 49-68. Iberjaca. Zaragoza.

GÓMEZ, C., SANAGUSTíN, M. V., SÁEZ, E. (2000): Población y recursos en Monegros: Análisis, diagnóstico y estrategias. Centro de Estudios sobre Despoblación y Desarrollo de Áreas Rurales.

GEORGE, P. (1980): Geografía Rural. Ariel. Barcelona.

HIGUERAS, A. (1996): «La population rurale espagnole». Espace, Polpulations, Societés No 1, pp. 103-110.

IAE. (1991): Población de derecho en relación con la actividad. Instituto Aragonés de Estadística. Diputación General de Aragón. Zaragoza.

IAE. (2001): Censos de población y viviendas. Indicadores de estructura demográfica. Instituto Aragonés de Estadística. Diputación General de Aragón. Zaragoza.

IAE. (2001): Movimiento Natural de la Población. Instituto Aragonés de Estadística. Diputación General de Aragón. Zaragoza.

IAE. (2002): Evolución intercensal (1900-2001) según los actuales términos municipales. Instituto Aragonés de Estadística. Diputación General de Aragón. Zaragoza.

IAE. (2003): Movimientos Migratorios. Instituto Aragonés de Estadística. Diputación General de Aragón. Zaragoza.

IAE. (2004): Evolución interanual (1985-2003), cifras padronales. Instituto Aragonés de Estadística. Diputación General de Aragón. Zaragoza.

INE (2006): Nomenclátor. Relaciones de unidades poblacionales en el año 2005.

INE., 1962-1972-1982-1989-1999. Censos Agrarios. Huesca, Zaragoza. Madrid.

MARÍN CANTALAPIEDRA, M. (1973): Población y recursos en la provincia de Zaragoza. CSIC. Zaragoza.

KOSTROWICKI, J. (1980): Geografía dell'Agricultura, Franco Angeli Editore, Milano.

MARTÍNEZ, A.M. (1983): «Los nuevos regadíos en Los Monegros», en Geografía de Aragón. Tomo 4, pp: 90-100. Guara Editorial. Zaragoza.

MAPA. (2001): Resultados técnico-económicos de explotaciones agrícolas de Aragón en 2000. Dirección General de Planificación Económica y Coordinación Institucional. Madrid.

MAPA. (2002): Resultados técnico-económicos de explotaciones agrícolas de Aragón en 2001. Dirección General de Planificación Económica y Coordinación Institucional. Madrid.

MINISTERIO DEL MEDIO AMBIENTE (2000): Libro Blanco del Agua en España. Madrid.

ORTEGA, N. (1979): Política agraria y dominación del espacio: Orígenes, caracterización y resultados de la política de colonización planteada en la España posterior a la guerra civil. Editorial Ayuso. Madrid.

ROMERO, S., 2005: Estructura productiva de las comarcas aragonesas: VAB comarcal 1999-2001. IAE. Gobierno de Aragón. Zaragoza. 